
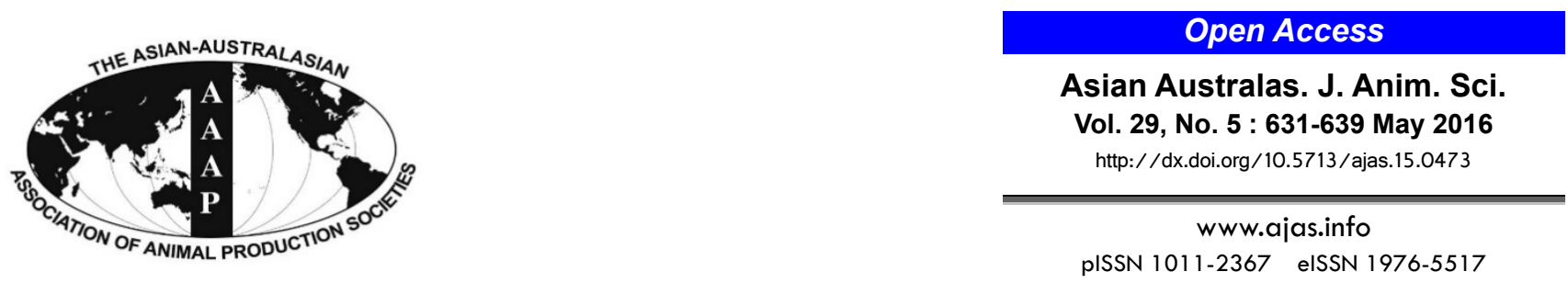

\title{
Complete Mitochondrial Genome Sequences of Chinese Indigenous Sheep with Different Tail Types and an Analysis of Phylogenetic Evolution in Domestic Sheep
}

\author{
Hongying Fan ${ }^{1,2, a}$, Fuping $\mathrm{Zhao}^{2, \mathrm{a}}$, Caiye $\mathrm{Zhu}^{2}$, Fadi Li ${ }^{3, *}$, Jidong Liu${ }^{4}$, \\ Li Zhang ${ }^{2}$, Caihong Wei ${ }^{2}$, and Lixin $\mathrm{Du}^{1,2, *}$ \\ ${ }^{1}$ College of Animal Science and Technology, Gansu Agricultural University, Lanzhou, Gansu 730070, China
}

\begin{abstract}
China has a long history of sheep (Ovis aries [O. aries]) breeding and an abundance of sheep genetic resources. Knowledge of the complete $O$. aries mitogenome should facilitate the study of the evolutionary history of the species. Therefore, the complete mitogenome of $O$. aries was sequenced and annotated. In order to characterize the mitogenomes of 3 Chinese sheep breeds (Altay sheep [AL], Shandong large-tailed sheep [SD], and small-tailed Hulun Buir sheep [sHL]), 19 sets of primers were employed to amplify contiguous, overlapping segments of the complete mitochondrial DNA (mtDNA) sequence of each breed. The sizes of the complete mitochondrial genomes of the sHL, AL, and SD breeds were 16,617 bp, 16,613 bp, and 16,613 bp, respectively. The mitochondrial genomes were deposited in the GenBank database with accession numbers KP702285 (AL sheep), KP981378 (SD sheep), and KP981380 (sHL sheep) respectively. The organization of the 3 analyzed sheep mitochondrial genomes was similar, with each consisting of 22 tRNA genes, 2 rRNA genes (12S rRNA and 16S rRNA), 13 protein-coding genes, and 1 control region (D-loop). The NADH dehydrogenase subunit 6 (ND6) and 8 tRNA genes were encoded on the light strand, whereas the rest of the mitochondrial genes were encoded on the heavy strand. The nucleotide skewness of the coding strands of the 3 analyzed mitogenomes was biased toward A and $\mathrm{T}$. We constructed a phylogenetic tree using the complete mitogenomes of each type of sheep to allow us to understand the genetic relationships between Chinese breeds of $O$. aries and those developed and utilized in other countries. Our findings provide important information regarding the $O$. aries mitogenome and the evolutionary history of $O$. aries inside and outside China. In addition, our results provide a foundation for further exploration of the taxonomic status of O. aries. (Key Words: Mitochondrial DNA, Mitogenome, Sheep, Phylogenetic, Mitochondrial Control Region)
\end{abstract}

\section{INTRODUCTION}

The origins of domestic sheep (Ovis aries [O. aries]) breeds remain a controversial topic. Domestic sheep can

\footnotetext{
* Corresponding Author: Fadi Li. Tel: +86-931-763-1225; Fax: +86-931-763-2468, E-mail: lifd@1zu.edu.cn / Lixin Du. Tel: +8610-6281-8815, Fax: +86-10-6281-8815, E-mail: 1xdu@263.net

2 Institute of Animal Sciences, Chinese Academy of Agricultural Sciences, Beijing 100193, China.

3 College of Pastoral Agriculture Science and Technology, Lanzhou University, Lanzhou, Gansu 730020, China.

4 Institute of Grass Ecology Research, Hulunbuir College, Hulunbuir 021008, Inner Mongolia Autonomous Region, China.

a These authors contributed equally to this work.

Submitted May 30, 2015; Revised Jul. 23, 2015; Accepted Aug. 12, 2015
}

interbreed with mouflon (Ovis orientalis orientalis), urial (Ovis orientalis vignei), and argali (Ovis ammon), complicating study of the origins of sheep breeds (Guo et al., 2005). China has a centuries-old tradition of sheep domestication, production, and breeding (Ma et al., 2006). In China, sheep are an important source of meat, wool, skin, and dairy products that fulfill agricultural, economic, cultural, and religious roles (Chen et al., 2006). At least 42 sheep breeds are indigenous to China (Resources, 2011); however, the origins and phylogenic relationships of these breeds are uncertain.

Evaluation of the structure and function of mitochondrial DNA (mtDNA) provides information useful in the study of molecular evolution, classification, population genetic analysis, and relationship identification. 
Analyses of mtDNA have been used to illuminate the maternal origins of populations (Bruford et al., 2003). Animal mtDNA is a small extrachromosomal genome that is typically 15 to $20 \mathrm{~kb}$ in size. With few exceptions, all animal mitochondrial genomes contain the same 37 genes: 2 ribosomal RNAs (rRNAs), 13 protein-coding genes and 22 transfer RNAs (tRNAs) (Boore, 1999). Previous studies constructed phylogenetic trees using the control region sequence (which includes the D-loop) and mitochondrial cytochrome b (CYTB) gene (Pedrosa et al., 2005; Gu et al., 2007). The earliest studies of mtDNA variation in domestic $O$. aries breeds exposed 3 distinct lineages (Wood and Phua, 1996; Hiendleder et al., 1998a, b; Guo et al., 2005).

In this study, we evaluated $3 O$. aries breeds indigenous to China, the Altay sheep, Shandong large-tailed sheep, and small-tailed Hulun Buir sheep, which were of the fat-rump tailed, fat-tailed, and small-tailed varieties, respectively. We sequenced the complete mitogenomes of each of the 3 types of sheep and confirmed the mitochondrial genome structure. The primary features of the sheep mitogenome, including gene structure, gene arrangement, initiation codon, termination codon, and anticodon, were described and compared among the 3 types of sheep. The A/T-content of each mitogenome was calculated by DNA frequency analysis. The mtDNA D-loop region and CYTB gene have been widely used to investigate the origins of sheep (Xin et al., 2006; Wang et al., 2007). However, the mtDNA D-loop region is best-suited to studies of the phylogeny of closely related breeds. Therefore, to facilitate the study of the evolutionary relationships of distantly related species, we analyzed the complete mitogenome. In addition, similar to other analyses of complete sheep mitogenomes, we performed a molecular phylogenetic analysis using the complete mitogenome and mtDNA control region via unweighted pair group method with arithmetic means (UPGMA) method (Tamura and Nei, 1993). This study will facilitate further investigations of the phylogenetic relationships of $O$. aries and provides important annotation information for the sheep mitogenome.

\section{MATERIALS AND METHODS}

\section{Sample collection and DNA extraction}

Blood samples were collected from Altay sheep (AL) from Fuhai County in the Xinjiang Uygur Autonomous Region, Shandong large-tailed sheep (SD) from Liaocheng City in Shandong Province, and small-tailed Hulun Buir sheep (sHL) from the Autonomous County of Evenki in the Inner Mongolia Autonomous Region. The samples were collected only from purebred, healthy, young female sheep.

Blood samples (approximately $10 \mathrm{cc}$ ) were drawn from the jugular veins of 40 unrelated domestic sheep into K3 ethylenediaminetetraacetic acid vacuum tubes by licensed veterinarian experts. Total genomic DNA from each individual was extracted using the QIAGEN DNeasy Blood \& Tissue Kit (Qiagen, Hilden, Germany) and stored at $-20^{\circ} \mathrm{C}$.

\section{Polymerase chain reaction amplification and sequencing}

Based on alignments and comparisons of complete mitochondrial sequences of other sheep (Hiendleder et al., 1998a), 19 sets of primers were employed to amplify contiguous, overlapping segments of the mtDNA from each type of sheep (Table 1). Polymerase chain reaction (PCR)

Table 1. PCR primers used for the analysis of sheep mitochondrial genomes

\begin{tabular}{lllcc}
\hline No. & \multicolumn{1}{c}{ Forward $\left(5^{\prime}-3^{\prime}\right)$} & \multicolumn{1}{c}{ Reverse $\left(5^{\prime}-3^{\prime}\right)$} & Product length $(\mathrm{bp})$ & Tm $\left({ }^{\circ} \mathrm{C}\right)$ \\
\hline 1 & AACTTAAAGCAAGGCACT & TTTACTTGAGGAGGGTGA & 918 & 916 \\
2 & AAATGACAATTCCCAACC & GATGTAGGGAGAAAATAGTTAGATC & 997 & 54 \\
3 & ATGGTTGAGGCCGGAGCA & TCAGAGTATCGTCGTGGT & 1,060 & 545 \\
4 & ATGAGCCAAAATCCACTT & ATTTCTGAGCATTGACCG & 984 & 51 \\
5 & CAATTCCAGGTCGTTTAA & CTTATTAGTGCAAGGGTG & 971 & 51 \\
6 & TGAAACCATCAGCCTATT & TACGAAGTGTCAGTATCAGG & 958 & 54 \\
7 & TCCTAATTGTCTGCTTCT & ATAGTCAGGTTAGGGGTA & 985 & 51 \\
8 & TACCCCTAACCTGACTAT & CAAGTGCTATGTGGCTAA & 946 & 54 \\
9 & AGCTCAATTTGCCTTCGC & GAGGGTTTGGATGGTTAG & 940 & 52 \\
10 & TCATGCGCTTTCATCACT & TGTCTTGCTCGTCGTTTA & 1,127 & 532 \\
11 & CCACCTAGCATTCCTTCA & TTGCTGCTTTATACAGTTATGG & 1,255 & 54 \\
12 & TCCACCACAATCACAAGC & GGTAATAACTGTTGCTCCT & 950 & 55 \\
13 & ATTTGCGACAATAGCCACAG & GTTGATAATGATTCAGGGA & 55 \\
14 & GAACAACCAACCTCCCTA & TGTGACCCAGGTGCCTAT & 1,124 & 51 \\
15 & TAATAGCCAAAGGAGGAT & CTTAAACTTGTGCGAGGA & 1,163 & 49 \\
16 & ACTGGACTATTCTATTACTAA & GAACTTTTCGTTCAACTA & 4,198 & 55 \\
17 & CCAGTTAAGGTGGCAGAG & GTAATTGGGAGCACGAAG & 584 & 50 \\
18 & CCCACAAACATAAGAAAT & TTTGAAGGCTCTTGGTCT & & 51 \\
19 & CTATGGCCGTCTGAGGCCTG & TAAGCAAGGCGTTGTGAG & &
\end{tabular}

PCR, polymerase chain reaction; $\mathrm{Tm}$, temperature. 
amplification was performed using an ABI 9700 Thermocycler. Each PCR reaction was carried out in a $25-$ $\mu \mathrm{L}$ reaction volume consisting of $12.5 \mu \mathrm{L}$ premixed enzyme, $2.0 \mu \mathrm{L}$ DNA template, $1.0 \mu \mathrm{L}$ of each primer (10 ppm), and $8.5 \mu \mathrm{L}$ sterile deionized water. The PCR conditions were as follows: an initial denaturation at $94^{\circ} \mathrm{C}$ for $3 \mathrm{~min}$, followed by 35 cycles of $94^{\circ} \mathrm{C}$ for $30 \mathrm{~s}$ (denaturation), $60^{\circ} \mathrm{C}$ for $30 \mathrm{~s}$ (annealing), and $72^{\circ} \mathrm{C}$ for $1 \mathrm{~min}$ (extension), with a final extension at $72^{\circ} \mathrm{C}$ for $10 \mathrm{~min}$. Each sample of amplified DNA $(5 \mu \mathrm{L})$ was inspected by electrophoresis on a $1 \%$ agarose gel to confirm the length of the amplified fragment.

The second data set used in this study was 16 Chinese sheep and 13 foreign sheep from National Center for Biotechnology Information (NCBI) (Table 2). These data set were used to construct the phylogenetic tree about Chinese and foreign sheep.

\section{Genome organization and structure analysis}

All of the sequences were inspected and assembled

Table 2. List of complete sheep mitogenomes available in the NCBI GenBank database

\begin{tabular}{|c|c|c|c|}
\hline No. & Species & Genbank No. & $\begin{array}{l}\text { Length } \\
\text { (bp) }\end{array}$ \\
\hline 1 & Tashkurgan sheep & KF938337.1 & 16,618 \\
\hline 2 & Yecheng sheep & KF938338.1 & 16,618 \\
\hline 3 & Qira Black sheep & KF938326.1 & 16,617 \\
\hline 4 & Sunite sheep & KF938317.1 & 16,543 \\
\hline 5 & Bashbay sheep & KF938330.1 & 16,618 \\
\hline 6 & Bayinbuluke sheep & KF938331.1 & 16,618 \\
\hline 7 & Tan sheep & KF938336.1 & 16,618 \\
\hline 8 & Small tailed Han sheep & KF977847.1 & 16,617 \\
\hline 9 & Duolang sheep & KF938332.1 & 16,618 \\
\hline 10 & Kirghiz sheep & KF938334.1 & 16,617 \\
\hline 11 & Qinghai Tibetan sheep & KF938325.1 & 16,617 \\
\hline 12 & Ujimqin sheep & KF938319.1 & 16,620 \\
\hline 13 & Hetian sheep & KF938322.1 & 16,620 \\
\hline 14 & Baerchuke sheep & KF938321.1 & 16,620 \\
\hline 15 & Turfan Black sheep & KF938324.1 & 16,620 \\
\hline 16 & Lanzhou large-tailed sheep & KF938335.1 & 16,617 \\
\hline 17 & Sahelian sheep & KF977846.1 & 16,617 \\
\hline 18 & Finnsheep & KF938355.1 & 16,617 \\
\hline 19 & Kulunda sheep & KF938358.1 & 16,617 \\
\hline 20 & Andi sheep & KF938340.1 & 16,617 \\
\hline 21 & Oxford down sheep & KF938359.1 & 16,617 \\
\hline 22 & Swiniarka sheep & KF938349.1 & 16,617 \\
\hline 23 & Pramenka sheep & KF938347.1 & 16,617 \\
\hline 24 & Mountain carpathian sheep & KF938357.1 & 16,617 \\
\hline 25 & Djallonke sheep & KF977845.1 & 16,617 \\
\hline 26 & Karakul sheep & KF938348.1 & 16,618 \\
\hline 27 & Minxian Black Fur sheep & KF938318.1 & 16,620 \\
\hline 28 & Ammon hodgsoni sheep (mouflon) & JX101654.1 & 16,688 \\
\hline 29 & Ammon isolate sheep (argali) & HM236188.1 & 16,613 \\
\hline
\end{tabular}

NCBI, National Center for Biotechnology Information. using DNASTAR (DNASTAR, Inc., Madison, WI, USA) and DNAMAN 7.0 (Lynnon LLC., San Ramon, CA, USA) software, after which the target sequences were assessed by basic local alignment search tool (BLAST) searches of the NCBI database. Thirteen typical protein-coding genes and 2 rRNA genes were identified by BLAST searches of the NCBI database ( $\mathrm{Hu}$ and Gao, 2014). Twenty-two tRNA genes and anticodons were annotated by tRNAscan-SE Search Server v.1.21 online (http://lowelab.ucsc.edu/ tRNAscan-SE/) (Lowe and Eddy, 1997). Initiation and termination codons were identified by comparison of the obtained genomes with fully sequenced $O$. aries mitogenomes. The complete mitochondrial genome was annotated using the program Sequin (National Center for Biotechnology Information, Bethesda, MD, USA). A nucleotide structure chart was generated for the entire mitogenome using OGDRAW (http://ogdraw.mpimpgolm.mpg.de/). The A+T-content of the entire mitogenome was calculated using the EditSeq program included in the Lasergene software package (DNASTAR, Inc., USA), after which $\mathrm{C}, \mathrm{G}, \mathrm{A}$, and $\mathrm{T}$ frequencies were calculated. Skewness was evaluated using the following formulas: GCskew $=(\mathrm{G}-\mathrm{C}) /(\mathrm{G}+\mathrm{C})$ and AT-skew $=(\mathrm{A}-\mathrm{T}) /(\mathrm{A}+\mathrm{T})$.

\section{Phylogenetic analysis}

The complete mitogenomes of 16 Chinese sheep breeds and 13 foreign sheep breeds were obtained from the National Center for Biotechnology Information (Table 2) and used to construct a phylogenetic tree. Clustering analysis was performed using MEGA 6.0 software (Tamura et al., 2013).

\section{RESULTS}

\section{Genome organization and structure}

To characterize the mitochondrial genomes of sHL, AL, and SD sheep, 19 sets of primers were employed to amplify contiguous, overlapping segments of complete mtDNA from each breed. The complete mitochondrial genomes of AL, SD, and sHL sheep have been submitted to the NCBI GenBank database (accession numbers: KP981380 [sHL], KP702285 [AL], and KP981378 [SD]). The mitochondrial genomes of the sHL, AL, and SD breeds were circular molecules that were $16,617 \mathrm{bp}, 16,613 \mathrm{bp}$, and $16,613 \mathrm{bp}$ in size, respectively.

The mitochondrial genomes of the sHL, AL, and SD breeds were similar in size to the mitogenomes of other sheep breeds ( $\mathrm{Hu}$ and Gao, 2014). In addition, the gene arrangements and transcriptional directions of the mitochondrial genomes of the sHL, AL, and SD breeds were similar to those of typical $O$. aries mitogenomes (Hiendleder et al., 1998a, b; $\mathrm{Hu}$ and Gao, 2014). The mitochondrial genes and D-loop region arrangements of the 
mitochondrial genomes of the sHL, AL, and SD breeds were identical. The nucleotide structure of the sHL mitogenome is shown Figure 1.

The nucleotide skewness of the coding strands of the mitogenomes of the sHL, AL, and SD breeds (AT-skew $=$ 0.10 , GC-skew $=-0.33$ ) was biased toward A and $\mathrm{T}$ (Table 3). The A-T rich pattern of the mitogenomes of the sHL, AL, and SD breeds was typical of vertebrate mitogenomes (Mayfield and McKenna, 1978; Hiendleder et al., 1998b). The nucleotide composition of the coding strand was biased toward $\mathrm{T}$ and $\mathrm{A}$ in $\mathrm{C}$, similar to most $O$. aries mitogenomes (Jiang et al., 2013). In addition, some variation in the nucleotide skewness of the whole mitogenome and control region was identified among the sHL, AL, and SD breeds (Table 3).

Four gene overlaps were found, all of which were on the $\mathrm{H}$-strand. In addition, 17 intergenic spacers were detected, with 11 on the heavy strand (H-strand) and 6 on the light strand (L-strand). The overlaps were 1 to $40 \mathrm{bp}$ in size, whereas the intergenic spacers were 1 to $32 \mathrm{bp}$ in size (Table 4). The longest overlap (40 bp) was located between ATP synthase protein 8 (ATP8) and ATP synthase protein 6 (ATP6). The longest intergenic spacer (32 bp) occurred between tRNA-Asn and tRNA-Cys.

The sHL, AL, and SD mitogenomes each contained 13 protein-coding genes, mitochondrially encoded NADH dehydrogenase 1-6 (ND1-6), mitochondrially encoded $\mathrm{NADH}$ dehydrogenase 4L (ND4L), mitochondrially encoded cytochrome c oxidase I (CO1), mitochondrially encoded cytochrome c oxidase II (CO2), mitochondrially encoded cytochrome c oxidase III (CO3) and ATP6, ATP8, and CYTB. ND1-6 were encoded on the L-strand, whereas the other 12 genes were encoded on the H-strand (Table 4). The start codon of ND2, ND3, and ND5 was ATA, whereas the start of the other 10 genes was ATG. ND1, ND2, ATP6, COX3, ND3, and ND4 were inferred to end with an incomplete stop codon (T--), whereas the stop codon of the other genes was TAA. With the exceptions of COX2 and ATP8, the different A/T content of protein-coding genes is different from the other breeds. ATP8 had the highest A/T content $(69.15 \%, 69.15 \%$, and $69.15 \%$ in the $\mathrm{SHL}, \mathrm{AL}$, and SD breeds, respectively), whereas COX3 had the lowest $\mathrm{A} / \mathrm{T}$ content $(54.72 \%, 54.21 \%$, and $54.21 \%$ in the $\mathrm{sHL}, \mathrm{AL}$, and SD breeds, respectively).

The 22 tRNA encoded in the complete mitochondrial genome of the sHL, AL, and SD breeds formed a typical clover-leaf secondary structure, with the exception of tRNAser (AGN), in which the DHU arm formed a simple loop. The 22 tRNA genes ranged in size from 66 to $75 \mathrm{bp}$. Fourteen of the tRNA genes were encoded on the H-strand, whereas 8 tRNA genes were encoded on the L-strand (Table 4). With the exceptions of tRNA-Asn, tRNA-Asp, tRNA-

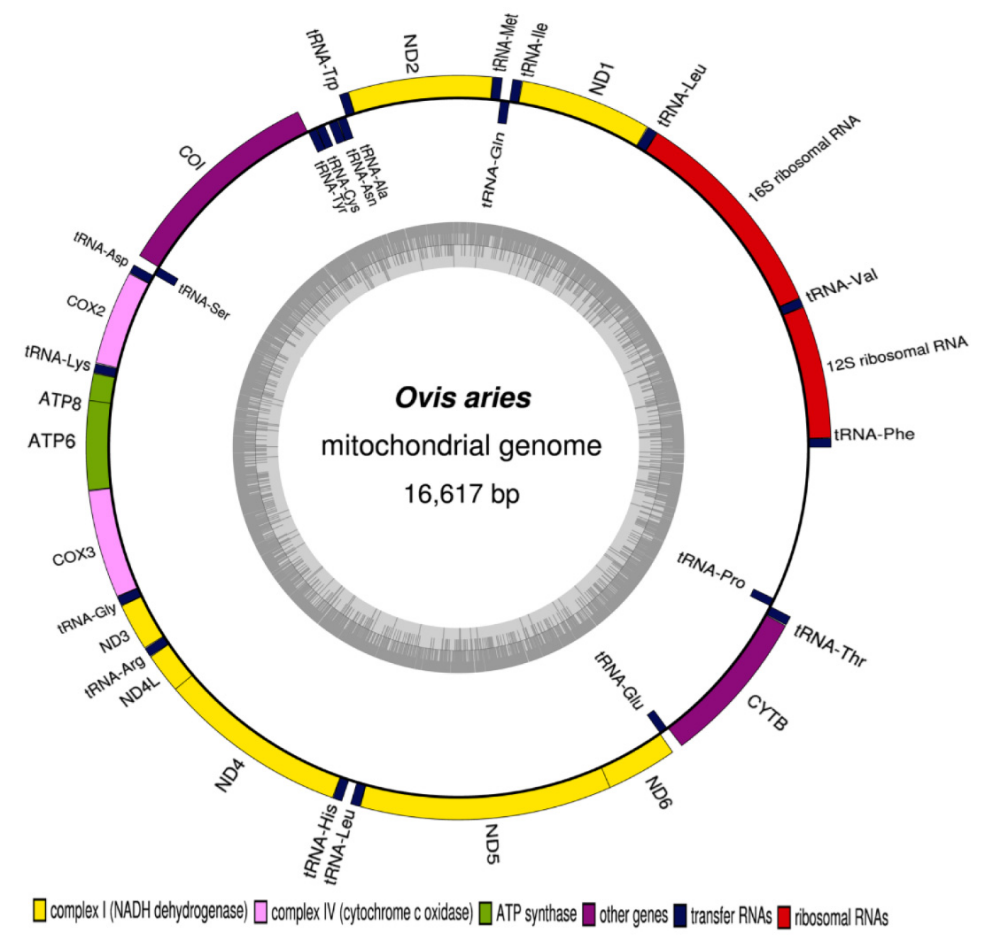

Figure 1. Mitochondrial gene arrangements of Ovis aries. Gene organization of the small-tailed Hulun Buir sheep for instance. Different color areas represent 13 protein-coding, 22 tRNA genes, 12S and16S rRNA genes and D-loop region. All protein-coding genes are encoded on the H-strand, with the exception of ND6 which is encoded on the L-strand. The two ribosomal RNA genes are encoded on the H-strand. Transfer RNA genes are designated by single-letter amino acid codes. Transfer RNA genes encoded on the H-strand and Lstrand are shown outside and inside the circular gene map, respectively. 
Table 3. Base compositions of sheep mitochondrial genomes

\begin{tabular}{|c|c|c|c|c|c|c|c|}
\hline \multirow{2}{*}{ Region } & \multirow{2}{*}{$\frac{\mathrm{A} / \mathrm{T} \text { content }(\%)}{\mathrm{sHL} / \mathrm{AL} / \mathrm{SD}}$} & \multicolumn{4}{|c|}{ Base composition $(\%)$} & \multirow{2}{*}{ AT-skewness } & \multirow{2}{*}{ GC-skewness } \\
\hline & & A & $\mathrm{G}$ & $\mathrm{T}$ & $\mathrm{C}$ & & \\
\hline \multicolumn{8}{|l|}{ Protein-coding gene } \\
\hline ND1 & $59.37 / 60.00 / 60.00$ & $31.73 / 32.25 / 32.25$ & $11.31 / 10.89 / 10.89$ & $27.64 / 27.75 / 27.75$ & $29.32 / 29.11 / 29.11$ & & \\
\hline ND2 & $64.01 / 63.63 / 63.63$ & $37.14 / 36.37 / 37.04$ & $8.25 / 8.35 / 8.35$ & $26.87 / 26.58 / 26.58$ & $27.74 / 28.02 / 28.02$ & & \\
\hline $\mathrm{COI}$ & $59.48 / 59.35 / 59.35$ & $29.13 / 28.93 / 28.93$ & $16.12 / 16.44 / 16.44$ & $30.36 / 30.42 / 30.42$ & $24.40 / 24.21 / 24.21$ & & \\
\hline $\operatorname{COX} 2$ & $62.28 / 62.28 / 62.28$ & $35.23 / 35.09 / 35.09$ & $13.16 / 13.30 / 13.30$ & $27.05 / 27.19 / 27.19$ & $24.56 / 24.42 / 24.42$ & & \\
\hline ATP8 & $69.15 / 69.15 / 69.15$ & $41.79 / 41.79 / 41.79$ & $5.97 / 5.97 / 5.79$ & $27.36 / 27.36 / 27.36$ & $24.88 / 24.88 / 24.88$ & & \\
\hline ATP6 & $60.09 / 59.65 / 59.65$ & $31.52 / 31.52 / 31.52$ & $11.78 / 11.63 / 11.63$ & $28.57 / 28.13 / 28.13$ & $28.13 / 28.72 / 28.72$ & & \\
\hline $\mathrm{COX} 3$ & $54.72 / 54.21 / 54.21$ & $26.15 / 25.64 / 25.64$ & $15.31 / 15.56 / 15.56$ & $28.57 / 28.57 / 28.57$ & $29.97 / 30.23 / 30.23$ & & \\
\hline ND3 & $57.80 / 58.38 / 58.38$ & $30.64 / 30.64 / 30.64$ & $11.85 / 11.85 / 11.85$ & $27.17 / 27.75 / 27.75$ & $30.35 / 29.77 / 29.77$ & & \\
\hline ND4L & $60.27 / 60.61 / 60.61$ & $30.30 / 30.30 / 30.30$ & $12.46 / 12.46 / 12.46$ & $29.97 / 30.30 / 30.30$ & $27.27 / 26.94 / 26.94$ & & \\
\hline ND4 & $60.96 / 60.81 / 60.81$ & $31.93 / 32.00 / 32.00$ & $10.74 / 10.67 / 10.67$ & $29.03 / 28.81 / 28.81$ & $28.30 / 28.52 / 28.52$ & & \\
\hline ND5 & $61.18 / 61.07 / 61.07$ & $33.77 / 33.66 / 33.66$ & $10.21 / 10.16 / 10.16$ & $27.40 / 27.40 / 27.40$ & $28.61 / 28.78 / 28.78$ & & \\
\hline ND6 & $64.96 / 64.77 / 64.77$ & $42.99 / 42.99 / 42.99$ & $7.01 / 6.82 / 6.82$ & $21.97 / 21.78 / 21.78$ & $28.03 / 28.41 / 28.41$ & & \\
\hline Cyt b & $58.68 / 58.60 / 58.60$ & $31.49 / 31.40 / 31.40$ & $12.89 / 12.98 / 12.98$ & $27.19 / 27.19 / 27.19$ & $28.42 / 28.42 / 28.42$ & & \\
\hline \multicolumn{8}{|l|}{ tRNA gene } \\
\hline tRNA-Phe & $61.76 / 61.76 / 61.76$ & $36.76 / 36.76 / 36.76$ & $19.12 / 19.12 / 19.12$ & $25.00 / 25.00 / 25.00$ & $19.12 / 19.12 / 19.12$ & & \\
\hline tRNA-Val & $70.15 / 70.15 / 70.15$ & $37.31 / 37.31 / 37.31$ & $13.43 / 13.43 / 12.43$ & $32.84 / 32.84 / 32.84$ & $16.42 / 16.42 / 16.42$ & & \\
\hline tRNA-Leu & $60.00 / 60.00 / 60.00$ & $32.00 / 32.00 / 32.00$ & $17.33 / 17.33 / 17.33$ & $28.00 / 28.00 / 28.00$ & $22.67 / 22.67 / 22067$ & & \\
\hline tRNA-Ile & 73.91/73.91/73.91 & $39.13 / 39.13 / 39.13$ & $15.94 / 15.94 / 15.94$ & $34.78 / 34.78 / 34.78$ & $10.14 / 10.14 / 1014$ & & \\
\hline tRNA-Gln & 61.11/61.11/61.11 & $36.11 / 36.11 / 36.11$ & $9.72 / 9.72 / 9.72$ & $25.00 / 25.00 / 25.00$ & $29.17 / 29.17 / 29.17$ & & \\
\hline tRNA-Met & $55.07 / 55.07 / 55.07$ & $27.54 / 27.54 / 27.54$ & $18.84 / 18.84 / 18.84$ & $27.54 / 27.54 / 27.54$ & $26.09 / 26.09 / 26.09$ & & \\
\hline tRNA-Trp & $64.18 / 64.18 / 64.18$ & $37.31 / 37.31 / 37.31$ & $16.42 / 16.42 / 16.42$ & $26.87 / 26.87 / 26.87$ & $19.40 / 19.40 / 19.40$ & & \\
\hline tRNA-Ala & $66.67 / 66.67 / 66.67$ & $39.13 / 39.13 / 39.13$ & $10.14 / 10.14 / 10.14$ & $27.54 / 27.54 / 27.54$ & $23.19 / 23.19 / 23.19$ & & \\
\hline tRNA-Asn & $54.79 / 56.16 / 56.16$ & $31.51 / 31.51 / 31.51$ & $15.07 / 15.07 / 15.07$ & $23.29 / 24.66 / 24.66$ & $30.14 / 28.77 / 28.77$ & & \\
\hline tRNA-Cys & $58.82 / 58.82 / 58.82$ & $27.94 / 27.94 / 27.94$ & $19.12 / 19.12 / 19.12$ & $30.88 / 30.88 / 30.88$ & $22.06 / 22.06 / 22.06$ & & \\
\hline tRNA-Tyr & $66.18 / 66.18 / 66.18$ & $29.41 / 29.41 / 29.41$ & $14.71 / 14.71 / 14.71$ & $36.76 / 36.76 / 36.76$ & $19.12 / 19.12 / 19.12$ & & \\
\hline tRNA-Ser & $57.97 / 57.97 / 57.97$ & $33.33 / 33.33 / 33.33$ & $15.94 / 15.94 / 15.94$ & $24.64 / 24.64 / 24.64$ & $26.09 / 26.09 / 26.09$ & & \\
\hline tRNA-Asp & $67.65 / 67.65 / 66.67$ & $39.71 / 39.71 / 39.13$ & $14.71 / 14.71 / 14.49$ & $27.94 / 27.94 / 27.54$ & $17.65 / 17.65 / 18.84$ & & \\
\hline tRNA-Lys & $66.18 / 63.24 / 63.24$ & $33.82 / 30.88 / 30.88$ & $14.71 / 17.65 / 17.65$ & $32.35 / 32.35 / 32.35$ & $19.12 / 19.12 / 19.12$ & & \\
\hline tRNA-Gly & $66.67 / 66.67 / 66.67$ & $33.33 / 33.33 / 33.33$ & $14.49 / 14.49 / 14.49$ & $33.33 / 33.33 / 33.33$ & $18.84 / 18.84 / 18.84$ & & \\
\hline tRNA-Arg & $74.63 / 74.63 / 75.00$ & $40.30 / 40.30 / 39.41$ & $11.94 / 11.94 / 11.76$ & $34.33 / 34.33 / 35.29$ & $13.43 / 13.43 / 13.24$ & & \\
\hline tRNA-His & 73.91/73.91/73.91 & $39.13 / 39.13 / 39.13$ & $13.04 / 13.04 / 13.04$ & $34.78 / 34.78 / 34.78$ & $13.04 / 13.04 / 13.04$ & & \\
\hline tRNA-Ser & $62.30 / 63.93 / 63.93$ & $32.79 / 34.43 / 34.43$ & $16.39 / 14.75 / 14.75$ & $29.51 / 29.51 / 29.51$ & $21.31 / 21.31 / 21.31$ & & \\
\hline tRNA-Leu & $67.61 / 67.61 / 67.61$ & $39.44 / 39.44 / 39.44$ & $16.90 / 16.90 / 16.90$ & $28.17 / 28.17 / 28.17$ & $15.49 / 15.49 / 15.49$ & & \\
\hline tRNA-Glu & $65.22 / 65.22 / 65.22$ & $39.13 / 39.13 / 39.13$ & $11.59 / 11.59 / 11.59$ & $26.09 / 26.09 / 26.09$ & $23.19 / 23.19 / 23.19$ & & \\
\hline tRNA-Thr & $60.00 / 60.00 / 60.00$ & $35.71 / 35.71 / 35.71$ & $17.14 / 17.14 / 17.14$ & $24.29 / 24.29 / 24.29$ & $22.86 / 22.86 / 22.86$ & & \\
\hline tRNA-Pro & $57.58 / 57.58 / 57.58$ & $34.85 / 34.85 / 34.85$ & $13.64 / 13.64 / 13.64$ & $22.73 / 22.73 / 22.73$ & $28.79 / 28.79 / 28.79$ & & \\
\hline 12SrRNA & $59.08 / 59.08 / 59.08$ & $36.85 / 36.85 / 36.85$ & $17.75 / 17.75 / 17.75$ & $22.23 / 22.23 / 22.23$ & $23.17 / 23.17 / 23.17$ & & \\
\hline 16SrRNA & $62.19 / 62.19 / 62.19$ & $37.43 / 37.43 / 37.43$ & $17.06 / 17.06 / 17.06$ & $24.76 / 24.76 / 24.76$ & $20.75 / 20.75 / 20.75$ & & \\
\hline D-loop & $62.49 / 62.63 / 62.63$ & $32.85 / 33.05 / 33.05$ & $14.56 / 14.41 / 14.41$ & $29.64 / 29.58 / 29.58$ & $22.95 / 22.97 / 22.97$ & & \\
\hline Entire mitogenome & $61.04 / 60.09 / 61.08$ & $33.65 / 33.61 / 33.66$ & $13.14 / 13.14 / 13.09$ & $25.82 / 25.87 / 25.83$ & $27.39 / 27.38 / 27.42$ & $0.10 / 0.10 / 0.10$ & $-0.33 /-0.33 /-0.33$ \\
\hline
\end{tabular}

sHL, small-tailed Hulun Buir sheep; AL, Altay sheep; SD, Shandong large-tailed sheep.

Lys, and tRNA-Arg, the tRNAs have equal $\mathrm{A} / \mathrm{T}$ content in the sHL, AL, and SD breeds. tRNA-Arg had the highest A/T content $(74.63 \%, 74.63 \%$, and $75.00 \%$ in the sHL, AL, and SD breeds, respectively), whereas tRNA-Asn had the lowest $\mathrm{A} / \mathrm{T}$ content $(54.79 \%, 56.16 \%$, and $56.16 \%$ in the sHL, AL, and SD breeds, respectively). In the sHL, AL, and SD breeds, the A/T content of the $16 \mathrm{~S}$ rRNA was $59.08 \%$, whereas that of the $12 \mathrm{~S}$ rRNA was $62.19 \%$. These findings provide an important foundation for further exploration of the taxonomic status of $O$. aries.

\section{Phylogenetic analysis}

We constructed a phylogenetic tree using the complete mtDNA (including the control region) of 32 breeds of sheep via the UPGMA method (Tamura et al., 1993). The complete dataset for the 32 sheep breeds included 2 parts: one part was from our sequencing data, whereas the other part was downloaded from the NCBI. The bootstrap consensus tree inferred from 1,000 replicates was taken to represent the taxonomy of the analyzed sheep breeds (Felsenstein, 1985). Branches corresponding to partitions reproduced in less than $50 \%$ of bootstrap replicates were collapsed. The evolutionary distances were computed using the maximum composite likelihood method (Tamura et al., 2004) and provided as the number of base substitutions per site. The analysis involved 32 nucleotide sequences. First, second, third, and noncoding codon positions were included. All positions containing gaps and missing data were 
Table 4. Summary of sheep mitochondrial genomes

\begin{tabular}{|c|c|c|c|c|c|c|c|c|}
\hline & \multirow{2}{*}{$\begin{array}{c}\text { Length (bp) } \\
\text { sHL/AL/SD }\end{array}$} & \multicolumn{2}{|c|}{ Position } & \multicolumn{2}{|c|}{ Codons } & \multirow{2}{*}{ Anticodon } & \multirow{2}{*}{$\begin{array}{l}\text { Intergenic } \\
\text { nucleotide }\end{array}$} & \multirow{2}{*}{ Strand } \\
\hline & & Start & End & Start & Stop & & & \\
\hline tRNA-Phe & $68 / 68 / 68$ & $1 / 1 / 1$ & $68 / 68 / 68$ & & & GAA & 0 & $\mathrm{H}$ \\
\hline rRNA & $958 / 958 / 958$ & $69 / 69 / 69$ & $1,026 / 1,026 / 1,026$ & & & & 0 & $\mathrm{H}$ \\
\hline tRNA-Val & $67 / 67 / 67$ & $1,027 / 1,027 / 1,027$ & $1,093 / 1,093 / 1,093 / 1,093$ & & & TAC & 0 & $\mathrm{H}$ \\
\hline rRNA & $1,574 / 1,571 / 1,571$ & $1,094 / 1,094 / 1,094$ & $2,667 / 2,664 / 2,664$ & & & & 0 & $\mathrm{H}$ \\
\hline tRNA-Leu & $75 / 75 / 75$ & $2668 / 2665$ & $2,742 / 2,739 / 2,739$ & & & TAA & 2 & $\mathrm{H}$ \\
\hline ND1 & $955 / 955 / 955$ & $2745 / 2742 / 2742$ & $3,699 / 3,696 / 3,696$ & ATG & T-- & & 1 & $\mathrm{H}$ \\
\hline tRNA-Ile & $69 / 69 / 69$ & $3701 / 3698 / 3698$ & $3,769 / 3,766 / 3,766$ & & & GAT & -1 & $\mathrm{H}$ \\
\hline tRNA-Gln & $72 / 72 / 72$ & $3767 / 3764 / 3764$ & $3,838 / 3,835 / 3,835$ & & & TTG & 2 & $\mathrm{~L}$ \\
\hline tRNA-Met & $69 / 69 / 69$ & $3841 / 3838 / 3838$ & $3,909 / 3,906 / 3,906$ & & & CAT & 0 & $\mathrm{H}$ \\
\hline ND2 & $1,042 / 1,042 / 1,042$ & $3910 / 3907 / 3907$ & $4,951 / 4,948 / 4,948$ & ATA & T-- & & 0 & $\mathrm{H}$ \\
\hline tRNA-Trp & $67 / 67 / 67$ & $4952 / 4949 / 4949$ & $5,018 / 5,015 / 5,015$ & & & TCA & 1 & $\mathrm{H}$ \\
\hline tRNA-Ala & $69 / 69 / 69$ & $5020 / 5017 / 5017$ & $5,088 / 5,085 / 5,085$ & & & TGC & 1 & $\mathrm{~L}$ \\
\hline tRNA-Asn & $73 / 73 / 73$ & $5090 / 5087 / 5087$ & $5,162 / 5,159 / 5,159$ & & & GTT & 32 & $\mathrm{~L}$ \\
\hline tRNA-Cys & $68 / 68 / 68$ & $5195 / 5192 / 5192$ & $5,262 / 5,259 / 5,259$ & & & GCA & 0 & $\mathrm{~L}$ \\
\hline tRNA-Tyr & $68 / 68 / 68$ & $5263 / 5260 / 5260$ & $5,330 / 5,327 / 537$ & & & GTA & 1 & $\mathrm{~L}$ \\
\hline COI & $1,545 / 1,545 / 1,545$ & $5332 / 5329 / 5329$ & $6,876 / 6,873 / 6,873$ & ATG & TAA & & -1 & $\mathrm{H}$ \\
\hline tRNA-Ser & $69 / 69 / 69$ & $6874 / 6871 / 6871$ & $6,942 / 6,939 / 6,939$ & & & TGA & 7 & $\mathrm{~L}$ \\
\hline tRNA-Asp & $68 / 68 / 68$ & $6950 / 6947 / 6947$ & $7,017 / 7,014 / 7,014$ & & & GTC & 1 & $\mathrm{H}$ \\
\hline $\mathrm{COX} 2$ & $684 / 684 / 684$ & 7019/7016/7016 & $7,702 / 7,699 / 7,699$ & ATG & TAA & & 3 & $\mathrm{H}$ \\
\hline tRNA-Lys & $68 / 68 / 68$ & 7706/7703/7703 & $7,773 / 7,770 / 7,770$ & & & TTT & 1 & $\mathrm{H}$ \\
\hline ATP8 & $201 / 201 / 201$ & 7775/7772/7772 & $7,975 / 7,972 / 7,972$ & ATG & TAA & & -40 & $\mathrm{H}$ \\
\hline ATP6 & $679 / 679 / 679$ & 7936/7933/7933 & $8,614 / 8,611 / 8,611$ & ATG & T-- & & 1 & $\mathrm{H}$ \\
\hline $\mathrm{COX} 3$ & $784 / 784 / 784$ & $8616 / 8613 / 8613$ & 9,399/9,396/9,396 & ATG & T-- & & 0 & $\mathrm{H}$ \\
\hline tRNA-Gly & $69 / 69 / 69$ & 9400/9397/9397 & $9,468 / 9,465 / 9,465$ & & & TCC & 0 & $\mathrm{H}$ \\
\hline ND3 & $346 / 346 / 346$ & $9469 / 9466 / 9466$ & $9,814 / 9,811 / 9,811$ & ATA & T-- & & 2 & $\mathrm{H}$ \\
\hline tRNA-Arg & $67 / 67 / 67$ & 9817/9814/9814 & $9,883 / 9,880 / 9,880$ & & & TCG & 1 & $\mathrm{H}$ \\
\hline ND4L & $297 / 297 / 297$ & 9885/9882/9882 & $10,181 / 10,178 / 10,178$ & ATG & TAA & & 7 & $\mathrm{H}$ \\
\hline ND4 & $1,378 / 1,378 / 1,378$ & $10,175 / 10,172 / 10,172$ & $11,552 / 11,549 / 11,549$ & ATG & T-- & & 0 & $\mathrm{H}$ \\
\hline tRNA-His & $69 / 69 / 69$ & $11,553 / 11,550 / 11,550$ & $11,621 / 11,618 / 11,618$ & & & GTG & 0 & $\mathrm{H}$ \\
\hline tRNA-Ser & $61 / 61 / 61$ & $11,622 / 11,619 / 11,619$ & $11,682 / 11,679 / 11,679$ & & & GCT & 0 & $\mathrm{H}$ \\
\hline tRNA-Leu & $71 / 71 / 71$ & $11,683 / 11,680 / 11,680$ & $11,753 / 11,750 / 11,750$ & & & TAG & 0 & $\mathrm{H}$ \\
\hline ND5 & $1821 / 1821$ & $11,754 / 11,751 / 11,751$ & $13,574 / 13,571 / 13,571$ & ATA & TAA & & -17 & $\mathrm{H}$ \\
\hline ND6 & $528 / 528 / 528$ & $13,558 / 13,555 / 13,555$ & $14,085 / 14,082 / 14,082$ & ATG & TAA & & 0 & $\mathrm{~L}$ \\
\hline tRNA-Glu & $69 / 69 / 69$ & $14,086 / 14,083 / 14,083$ & $14,154 / 14,151 / 14,151$ & & & TTC & 4 & $\mathrm{~L}$ \\
\hline Cyt b & $1139 / 1139$ & $14,159 / 14,156 / 14,156$ & $15,298 / 15,295 / 15,295$ & ATG & TAA & & 3 & $\mathrm{H}$ \\
\hline tRNA-Thr & $70 / 70 / 70$ & $15,302 / 15,299 / 15,299$ & $15,371 / 15,368 / 15,368$ & & & TGT & 0 & $\mathrm{H}$ \\
\hline tRNA-Pro & $65 / 65 / 65$ & $15,371 / 15,368 / 15,368$ & $15,436 / 15,433 / 15,433$ & & & TGG & 0 & $\mathrm{~L}$ \\
\hline D-loop & $1,180 / 1,180 / 1,180$ & $15,437 / 15,434 / 13,434$ & $16,617 / 16,613 / 16,613$ & & & & & \\
\hline
\end{tabular}

sHL, small-tailed Hulun Buir sheep; AL, Altay sheep; SD, Shandong large-tailed sheep.

eliminated. There were a total of 16,530 positions in the final dataset. Clustering analyses were conducted using MEGA6.0 (Tamura et al., 2013).

Phylogenetic analysis of the complete mitogenome showed that the Chinese local sheep breeds were clustered into a single group, whereas the foreign breeds were closely clustered into another group (Figure 2). The mouflon $(O$. orientalis orientalis) (ammon_isolate sheep) and argali ( $O$. ammon) (ammon_hodgsoni sheep) were placed into a separate branch as outgroups. The phylogenetic tree constructed using the D-loop of the Chinese and foreign sheep breeds showed a disordered distribution (Figure 3). These results indicate that evolutionary analyses utilizing full mitogenomes can provide information regarding the relationships between distantly related species that cannot be generated by analysis of the D-loop region only.

\section{DISCUSSION}

The complete mitochondrial genomic DNA sequences 


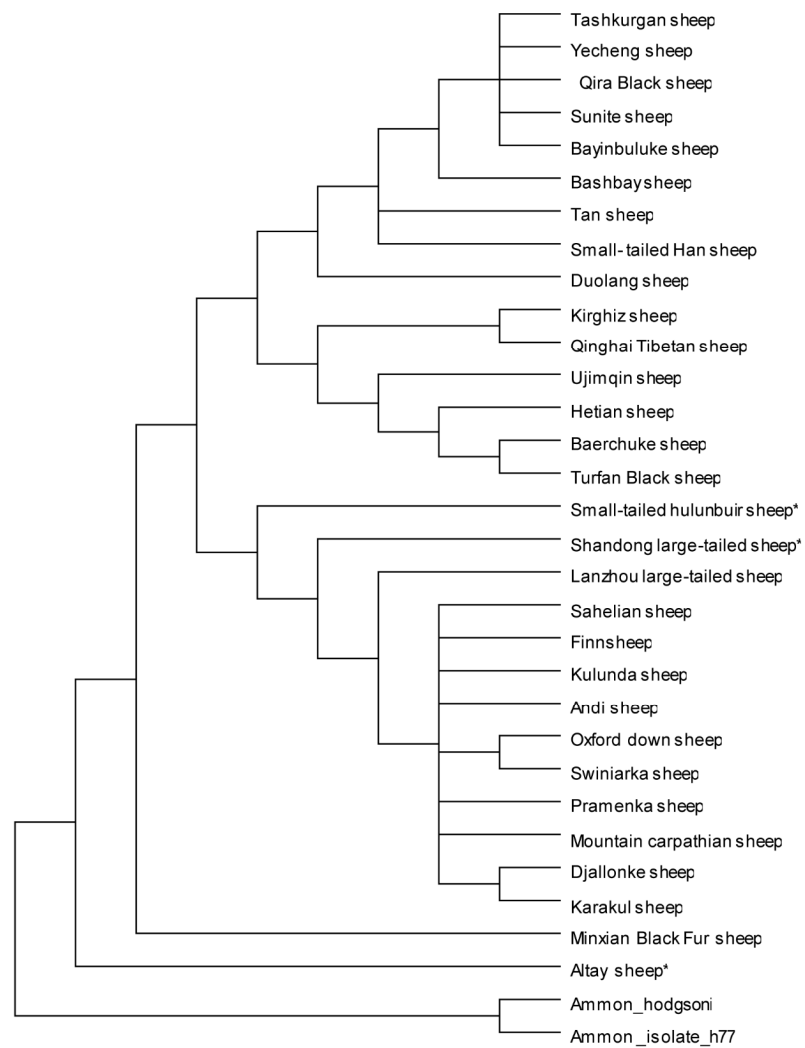

Figure 2. Evolutionary relationships of sheep based on the mitogenomes. Phylogenetic trees of Ovis aries constructed by the complete mitochondrial genome of 30 sheep. Ammon hodgsoni and Ammon isolate h77 were selected out groups. The evolutionary distances were computed using the Maximum Composite Likelihood method. Tree topologies was inferred using the UPGMA method. The asterisk indicates the sequence generated in this research.

of 3 breeds of indigenous Chinese sheep, AL, SD, and sHL, were 16,613 to $16,617 \mathrm{bp}$ in length and contained 13 protein-coding genes, 22 tRNA genes, 2 rRNA genes, and 1 putative control region. The organization and structure of the mitogenomes of the $\mathrm{AL}, \mathrm{SD}$, and sHL sheep were similar to those of other domestic sheep, including the Texel and Merinolandschaf breeds (Hiendleder et al., 1998a, b; $\mathrm{Hu}$ and Gao, 2014). The minor differences in the sizes of the genomes of domestic sheep were due primarily to changes in the control region.

The AL, SD, and sHL mitogenomes had similar A/T content (approximately $60 \%$ to $61 \%$ ), which was also similar to that of the Texel, Merino, and Merinolandschaf breeds (Hiendleder et al., 1998a, b; Lancioni et al., 2013; $\mathrm{Hu}$ and Gao, 2014). The gene overlaps and intergenic spacers among $1 \mathrm{bp}$ to $7 \mathrm{bp}$ at the large, other than tRNAAla and tRNA-Asn, ATP8, and ATP6, also ND5 and ND6 were $32,-40,-17$, respectively.

The relative organization of the 37 genes and control regions comprising the mitogenomes of the $\mathrm{sHL}, \mathrm{AL}$, and SD breeds was identical with that of other domestic sheep

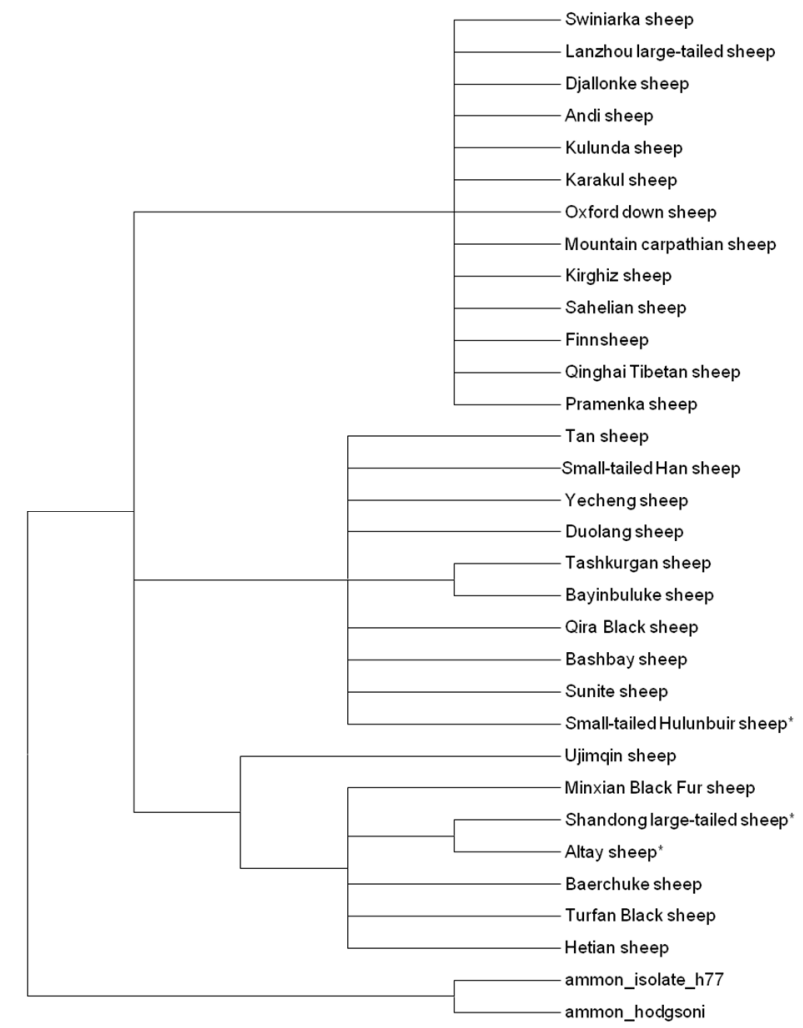

Figure 3. Evolutionary relationships of sheep based on the D-loop region. Results of phylogenetic analyses via UPGMA method using analysis indicated evolutionary relationships among 30 individuals based on mtDNA D-loop region. Ammon hodgsoni and Ammon isolate $\mathrm{h} 77$ were selected out groups. The evolutionary distances were computed using the Maximum Composite Likelihood method. The asterisk indicates the sequence generated in this research. UPGMA, unweighted pair group method with arithmetic means; mtDNA, mitochondrial DNA.

breeds (Hiendleder et al., 1998a, b; Hu and Gao, 2014). The 13 protein-coding genes in the mitogenomes of the sHL, $\mathrm{AL}$, and SD breeds began with initiation codons ATG or ATA, but only 3 genes (ND2, ND3, and ND5) used the latter. About half of the 13 protein-coding genes had incomplete termination codons, whereas the others had the typical TAA stop codon. Incomplete termination codon $(T)$ is in the process of transcription by TAA after the formation affixed Poly (A) terminal translated (Clayton, 1991). Incomplete stop codons are commonly found in many vertebrate mitochondrial genes (Wang et al., 2014).

The phylogenetic tree based on the mitogenome clusters the sHL and SD sheep into the same clade, suggesting they have closer relationship than AL. This agrees with the fact that both of SD and SHL are fat-tailed sheep while AL are fat-rump tailed sheep. In addition to details regarding the organization of the mitochondrial genome of indigenous Chinese sheep, this study provides information regarding the evolutionary relationship between the mitogenomes of indigenous Chinese sheep and foreign sheep. The NJ, ML, 
and UPGMA trees constructed according to the maximum composite likelihood method showed the same results; therefore, we showed only the UPGMA tree. Our phylogenetic tree, constructed using the entire mitogenome, clearly shows the evolutionary separation between indigenous Chinese sheep and foreign sheep, whereas the phylogenetic tree constructed using only the control region did not show this relationship.

\section{CONCLUSION}

The present study provides the complete mitochondrial genome DNA sequences of the small-tailed Hulun Buir sheep, Altay sheep, and Shandong large-tailed sheep, which are indigenous to China. The mitogenomes of the smalltailed Hulun Buir sheep, Altay sheep, and Shandong largetailed sheep were $16,617 \mathrm{bp}, 16,613 \mathrm{bp}$, and $16,613 \mathrm{bp}$ in length, respectively, and included a typical set of 37 genes: 13 protein-coding genes, 2 rRNA genes, and 22 tRNA genes, as well as a control region. The gene annotations and genome organizations of the mitogenomes of small-tailed Hulun Buir sheep, Altay sheep, and Shandong large-tailed sheep have been submitted to the GenBank database. Phylogenetic relationships determined via analysis of complete mitochondrial genomes may be more precise than those determined via analysis of only the mitochondrial control region.

\section{CONFLICT OF INTEREST}

We certify that there is no conflict of interest with any financial organization regarding the material discussed in the manuscript.

\section{ACKNOWLEDGMENTS}

This work was supported by the Agriculture Science and Technology Innovation Program (no. ASTIP-IAS-TS-6) and the National Modern Agricultural Industry Technology Fund for Scientists in Sheep Industry System (no. CARS39-04B).

\section{REFERENCES}

Boore, J. L. 1999. Animal mitochondrial genomes. Nucl. Acids Res. 27:1767-1780.

Bruford, M. W., D. G. Bradley, and G. Luikart. 2003. DNA markers reveal the complexity of livestock domestication. Nat. Rev. Genet. 4:900-910.

Chen, S. Y., Z. Y. Duan, T. Sha, J. Xiangyu, S. F. Wu, and Y. P. Zhang. 2006. Origin, genetic diversity, and population structure of Chinese domestic sheep. Gene 376:216-223.

Clayton, D. A 1991. Replication and transcription of vertebrate mitochondrial DNA. Annu. Rev. Cell Biol. 7:453-478.
Felsenstein, J. 1985. Confidence limits on phylogenies: An approach using the bootstrap. Evolution. 39:783-791.

Gu, Z., X. Zhao, N. Li, and C. Wu. 2007. Complete sequence of the yak (Bos grunniens) mitochondrial genome and its evolutionary relationship with other ruminants. Mol. Phylogenet. Evol. 42:248-255.

Guo, J., L. X. Du, Y. H. Ma, W. J. Guan, H. B. Li, Q. J. Zhao, X. $\mathrm{Li}$, and S. Q. Rao 2005. A novel maternal lineage revealed in sheep (Ovis aries). Anim. Genet. 36:331-336.

Hiendleder, S., H. Lewalski, R. Wassmuth, and A. Janke 1998a. The complete mitochondrial DNA sequence of the domestic sheep (Ovis aries) and comparison with the other major ovine haplotype. J. Mol. Evol. 47:441-448.

Hiendleder, S., K. Mainz, Y. Plante, and H. Lewalski. 1998b. Analysis of mitochondrial DNA indicates that domestic sheep are derived from two different ancestral maternal sources: No evidence for contributions from urial and argali sheep. J. Hered. 89:113-120.

Hu, X. D. and L. Z. Gao. 2014. The complete mitochondrial genome of domestic sheep, Ovis aries. Mitochondrial DNA 27:1425-1427.

Jiang, L., G. Wang, S. Tan, S. Gong, M. Yang, Q. Peng, R. Peng, and F. Zou 2013. The complete mitochondrial genome sequence analysis of Tibetan argali (Ovis ammon hodgsoni): Implications of Tibetan argali and Gansu argali as the same subspecies. Gene 521:24-31.

Lancioni, H., P. Di Lorenzo, S. Ceccobelli, U. A. Perego, A. Miglio, V. Landi, M. T. Antognoni, F. M. Sarti, E. Lasagna, and A. Achilli. 2013. Phylogenetic relationships of three Italian merino-derived sheep breeds evaluated through a complete mitogenome analysis. PLoS One 8:e73712.

Lowe, T. M. and S. R. Eddy. 1997. tRNAscan-SE: a program for improved detection of transfer RNA genes in genomic sequence. Nucl. Acids Res. 25:955-964.

Ma, Y.-H., S.-Q. Rao, S.-J. Lu, G.-Y. Hou, W.-J. Guan, H.-B. Li, X. Li, Q.-J. Zhao, and J. Guo. 2006. Phylogeography and Origin of Sheep Breeds in Northern China. Conserv. Genet. 7:117-127.

Mayfield, J. E. and J. F. McKenna. 1978. A-T rich sequences in vertebrate DNA. Chromosoma 67:157-163.

Pedrosa, S., M. Uzun, J. J. Arranz, B. Gutierrez-Gil, F. San Primitivo, and Y. Bayon. 2005. Evidence of three maternal lineages in Near Eastern sheep supporting multiple domestication events. Proc. Biol. Sci. 272:2211-2217.

Resources (China National Commission of Animal Genetic) 2011. Animal Genetic Resources in China: Sheep and Goats records: China Agriculture Press, Beijing, China.

Tamura, K. and M. Nei. 1993. Estimation of the number of nucleotide substitutions in the control region of mitochondrial DNA in humans and chimpanzees. Mol. Biol. Evol. 10:512526.

Tamura, K., M. Nei, and S. Kumar. 2004. Prospects for inferring very large phylogenies by using the neighbor-joining method. Proc. Natl. Acad. Sci. USA. 101:11030-11035.

Tamura, K., G. Stecher, D. Peterson, A. Filipski, and S. Kumar. 2013. MEGA6: Molecular Evolutionary Genetics Analysis version 6.0. Mol. Biol. Evol. 30:2725-2729.

Wang, X., Y. H. Ma, H. Chen, and W. J. Guan. 2007. Genetic and phylogenetic studies of Chinese native sheep breeds (Ovis 
aries) based on mtDNA D-loop sequences. Small Rumin. Res. Wood, N. J. and S. H. Phua. 1996. Variation in the control region 72:232-236.

Wang, X., Y. Q. Zhang, D. C. He, X. M. Yang, B. Li, D. C. Wang, J. Guang, F. Xu, J. Y. Li, X. Gao, H. J. Gao, L. P. Zhang, and X. Z. Zhang. 2014. The complete mitochondrial genome of Bos taurus coreanae (Korean native cattle). Mitochondrial DNA 27:120-121. sequence of the sheep mitochondrial genome. Anim. Genet. 27:25-33.

Xin, W., M. A. Yue-Hui, and C. Hong. 2006. Analysis of the genetic diversity and the phylogenetic evolution of Chinese sheep based on Cyt b gene sequences. Acta Genet. Sin. 33: 1081-1086. 